\title{
Identification of potential key genes in esophageal adenocarcinoma using bioinformatics
}

\author{
ZHIYU DONG, JUNWEN WANG, HAIQIN ZHANG, TINGTING ZHAN, YING CHEN and SHUCHANG XU \\ Department of Gastroenterology, Tongji Hospital, Tongji University School of Medicine, Shanghai 200065, P.R. China
}

Received December 23, 2017; Accepted November 7, 2018

DOI: $10.3892 /$ etm.2019.7973

\begin{abstract}
Esophageal adenocarcinoma (EAC) is the predominant pathological subtype of esophageal cancer in Europe and the USA. The present bioinformatics study analyzed a high-throughput sequencing dataset, GSE94869, to determine differentially expressed genes (DEGs) in order to identify key genes, biological processes and pathways associated with EAC. Functional enrichment analysis was performed using the Database for Annotation Visualization and Integrated Discovery. The co-expression network of the DEGs was established using Weighted Gene Co-Expression Network Analysis and visualized using Cytoscape. A Kaplan-Meier analysis based on The Cancer Genome Atlas (TCGA) database was used to identify prognosis-associated genes. Univariate and multivariate Cox proportional hazard models were used to identify genes with a prognostic value regarding relapse-free survival (RFS), while validation of the differential expression of prognosis-associated genes was performed using a box plot based on data from TCGA and another microarray dataset, GSE26886. A total of 130 DEGs, comprising 82 upregulated and 48 downregulated genes, were identified. The upregulated DEGs were significantly associated with extracellular matrix organization, disassembly, and the phosphoinositide-3 kinase/AKT, Rap1 and Ras signaling pathways, while the downregulated genes were associated with the Wnt signalling pathway. Subsequently, two co-expression modules
\end{abstract}

Correspondence to: $\mathrm{Dr}$ Shuchang $\mathrm{Xu}$, Department of Gastroenterology, Tongji Hospital, Tongji University School of Medicine, 389 Xincun Road, Putuo, Shanghai 200065, P.R. China

E-mail: xschang@163.com

Abbreviations: EAC, esophageal adenocarcinoma; WGCNA, Weighted Gene Co-Expression Network Analysis; DEG, differentially expressed gene; GO, Gene Ontology; KEGG, Kyoto Encyclopedia of Genes and Genomes; DAVID, Database for Annotation Visualization and Integrated Discovery; RFS, relapse-free survival

Key words: esophageal adenocarcinoma, differential expression genes, functional enrichment analysis, Weighted Gene Co-Expression Network Analysis, Kaplan-Meier analysis, Cox proportional hazards model were established and 20 hub genes were identified. The blue module was associated with the Rap1 signaling pathway, while the turquoise module was associated with the Ras and Rap1 signaling pathways. Among them, methyltransferase like 7B (METTL7B) was associated with RFS. Furthermore, the overexpression of METTL7B in EAC was successfully validated using data from TCGA and GSE26886. The present study identified key genes and provides potential biomarkers for the diagnosis and treatment of EAC.

\section{Introduction}

Esophageal cancer is one of the most common types of malignant tumor encountered in the clinic and is the 6th most frequent cause of cancer-associated mortality. Esophageal adenocarcinoma (EAC) is the predominant pathological subtype of esophageal cancer in Europe and the USA (1). Due to a lack of reliable early diagnostic methods and efficient intervention approaches, EAC has had a poor five-year survival rate of $<20 \%$ (2). Although significant progress has been made in the medical field over the past few years, the median survival time of EAC has only slightly increased (3). Therefore, more research effort is required to explore potential prognostic markers and therapeutic strategies for EAC.

In recent years, molecular biomarkers with an early diagnostic and prognostic value for EC have been investigated. Chen et al (4) performed a systematic review of the literature and summarized that cyclooxygenase- 2 and HER-2 are valuable prognostic markers for EAC. A study by Darlavoix et al (5) on EAC reported that the decrease in E-cadherin expression and the altered expression of CD44 and dickkopf WNT signaling pathway inhibitor 1 may indicate an increased risk of EAC progression. A large number of microRNA (miR) biomarkers have been identified; for instance, the loss of miR-31 is considered as an early molecular marker for the metaplasia-to-dysplasia transition of EAC, while miR-194, miR-196a and miR-375 are considered as oncogenic factors $(6,7)$. In spite of the large number of miR biomarkers identified, only few of them are suitable for clinical use due to a lack of sensitivity and specificity. An increased knowledge of EAC-associated biomarkers may provide further mechanistic insight and lay a foundation for novel approaches to prevent, diagnose and treat EAC.

High-throughput sequencing technology is a widely used tool to explore genetic alterations during tumorigenesis. In 
the present study, a high-throughput sequencing dataset was analysed using bioinformatics tools to identify differentially expressed genes (DEGs). The DEGs were subjected to functional enrichment, co-expression network analysis and survival analysis to identify key genes in EAC, while the validation of key gene expression levels in EAC and normal epithelial tissues was performed using data from The Cancer Genome Atlas (TCGA) database and another microarray dataset.

\section{Materials and methods}

High-throughput sequencing data. The GSE94869 gene expression profile obtained through high-throughput sequencing was downloaded from the Gene Expression Omnibus database (https://www.ncbi.nlm.nih.gov/geo/) $(8,9)$. The data]]set was based on the Illumina MiSeq [Homo sapiens (hsa)] platform (Illumina, Inc., San Diego, CA, USA). The GSE94869 dataset included 40 pairs of EAC and matched normal esophageal squamous epithelium tissues.

DEG analysis. The DEGs in EAC tissues compared with normal tissues were identified using the Linear Models for Microarray Analysis (Limma) package in R software (10). The Benjamini-Hochberg false discovery rate (FDR) was used to correct the P-values. An adjusted P-value of $<0.05$ and a fold change of $\geq 2$ were used as the cut-off criteria for the DEG analysis.

Functional enrichment analysis of DEGs. Gene Ontology (GO) (11) and the Kyoto Encyclopedia of Genes and Genomes (KEGG) (12) analyses are widely used to identify the most correlative GO terms in the category biological process (BP) and relevant pathway information, and were performed through the online tool Database for Annotation Visualization and Integrated Discovery (13). A corrected P-value (P-value adjusted using Benjamini-Hochberg) of $<0.05$ was used as the cut-off criterion.

Co-expression module detection and functional enrichment analysis for each module. The weighted gene co-expression network analysis (WGCNA) (14) was restricted to the DEGs by using the WGCNA package in R software. An unsupervised co-expression association was constructed on the basis of the adjacency matrix of connection strengths using Pearson's correlation coefficients. Gene modules that had a high topologic overlap were identified as gene sets and a minimum gene module size of 30 was used to cut branches. The top 10 genes with the highest network connectivity in each module were identified as hub genes. Subsequently, the co-expression network of each module was presented using Cytoscape 3.4.0 (15). GO and KEGG analyses for each module were then performed as specified above. A corrected P-value (P-value adjusted using Benjamini-Hochberg) of $<0.05$ was used as the cut-off criterion.

Survival analysis depending on hub genes. The mRNA transcript per million of 104 EAC tissue samples and corresponding follow-up information for these patients were downloaded from the Xena browser from the University of California Santa Cruz (https://xenabrowser. net/datapages/?host=https://tcga.xenahubs.net). The survival $\mathrm{R}$ package (https://CRAN.R-project.org/package=survival) (16) was used to explore the prognostic value of hub genes. Kaplan-Meier survival curves were plotted. Relapse-free survival (RFS) was used as the survival endpoint and only the 62 patients with RFS data were included. Patients with EAC were divided into low and high expression groups according to the median expression value of each hub gene. A Log-rank $\mathrm{P}$-value of $<0.05$ was considered to indicate statistical significance.

Cox proportional hazards $(\mathrm{CPH})$ analysis. The association of clinical parameters, including age at diagnosis, history of alcohol use or Barrett's esophagus, pathologic tumor-nodes-metastasis stage with survival was assessed using the univariate $\mathrm{CPH}$ model. The results of the CPH analysis were presented as hazard ratios, along with parameters of significance and $95 \%$ confidence intervals. All prognosis-associated genes identified by the Kaplan-Meier analysis were converted into ordinal or unordered categorical variables based on quartiles (Q) in order to determine the risk of recurrence of EAC for each quartile expression level of the prognosis-associated genes. A P-value for the trend referred to different quartiles was obtained from the $\mathrm{CPH}$ model when the expression of prognosis-associated genes was categorized into ordinal categorical variables. As for unordered categorical variables, the CPH analysis was applied with the lowest quartiles as a reference. Model 1 remained unadjusted and Model 2 was adjusted for risk factors that were indicated to have an effect $(\mathrm{P}<0.2)$ on survival in the univariate analysis. $\mathrm{P}<0.05$ was considered to indicate statistical significance.

Validation of differential expression of prognosis-associated genes. Validation of the expression levels of all prognosis-associated genes identified through Kaplan-Meier analysis was performed using a box plot to visualize the difference in expression between EAC and normal tissues based on data from TCGA and the dataset GSE26886 (17). The data of 8 EAC tissues and paired paracancerous lesions were obtained from the TCGA database. The GSE26886 dataset was based on the Affymetrix Human Genome U133 Plus 2.0 Array platform (Thermo Fisher Scientific, Inc., Waltham, MA, USA) and included the data of 21 EAC tissues and 19 normal esophageal squamous epithelial tissues. The expression levels were presented as the mean \pm standard deviation. A P-value of $<0.05$ was considered to indicate a statistically significant difference.

\section{Results}

DEG and functional enrichment analysis. In total, 130 DEGs, including 82 upregulated and 48 downregulated genes, were identified using the Limma package. The GO BP analysis demonstrates that the upregulated DEGs were significantly associated with extracellular matrix organization, endodermal cell differentiation and extracellular matrix disassembly, while the downregulated DEGs were significantly associated with cell fate commitment, neuron differentiation and the canonical Wnt signaling pathway. The KEGG pathway analysis demonstrated that the upregulated genes were significantly enriched in focal 
Table I. GO terms in the category biological process and Kyoto Encyclopedia of Genes and Genomes pathways significantly enriched by the differentially expressed genes in esophageal adenocarcinoma.

\begin{tabular}{clrl}
\hline Term/pathway & \multicolumn{1}{c}{ Description } & Enriched genes (n) & Adjusted P-value \\
\hline Upregulated & & & \\
GO:0030198 & Extracellular matrix organization & 12 & $9.43 \times 10^{-7}$ \\
GO:0035987 & Endodermal cell differentiation & 5 & $2.92 \times 10^{-3}$ \\
GO:0030168 & Platelet activation & 7 & $3.95 \times 10^{-3}$ \\
GO:0007596 & Blood coagulation & 8 & $4.23 \times 10^{-3}$ \\
GO:0022617 & Extracellular matrix disassembly & 6 & $4.33 \times 10^{-3}$ \\
GO:0048013 & Ephrin receptor signaling pathway & 6 & $6.57 \times 10^{-3}$ \\
hsa04510 & Focal adhesion & 13 & $7.58 \times 10^{-6}$ \\
hsa04151 & PI3K-Akt signaling pathway & 14 & $1.53 \times 10^{-4}$ \\
hsa04611 & Platelet activation & 8 & $1.69 \times 10^{-3}$ \\
hsa05200 & Pathways in cancer & 13 & $1.70 \times 10^{-3}$ \\
hsa04015 & Rap1 signaling pathway & 10 & $1.74 \times 10^{-3}$ \\
hsa04014 & Ras signaling pathway & 10 & $1.86 \times 10^{-3}$ \\
hsa05146 & Amoebiasis & 7 & $3.78 \times 10^{-3}$ \\
hsa04071 & Sphingolipid signaling pathway & 7 & $6.50 \times 10^{-3}$ \\
hsa04512 & ECM-receptor interaction & 6 & $9.85 \times 10^{-3}$ \\
Downregulated & & & $1.69 \times 10^{-3}$ \\
GO:0045165 & Cell fate commitment & 5 & $2.03 \times 10^{-3}$ \\
GO:0030182 & Neuron differentiation & $6.09 \times 10^{-3}$ \\
GO:0060070 & Canonical Wnt signaling pathway & 6 & $3.38 \times 10^{-3}$ \\
GO:0000122 & Negative regulation of transcription from RNA & 6 & $1.39 \times 10^{-5}$ \\
hsa04550 & polymerase II promoter & Signaling pathways regulating pluripotency of stem cells & $9.79 \times 10^{-3}$ \\
hsa04310 & Wnt signaling pathway & 9 & \\
\hline
\end{tabular}

GO, Gene Ontology; hsa, Homo sapiens; ECM, extracellular matrix; PI3K, phosphoinositide-3 kinase.

adhesion, the phosphoinositide-3 kinase (PI3K)-Akt signaling pathway and the Rap1 and Ras signaling pathways, while the downregulated genes were significantly associated with signaling pathways regulating pluripotency of stem cells and the Wnt signaling pathway. These results are presented in Table I.

Co-expression module detection and functional enrichment analysis. WGCNA was performed to construct gene co-expression modules from the expression profiles of the 130 DEG. A total of 2 modules comprising of 36 and 50 genes each were identified. The co-expression networks of the 2 modules including their hub genes are visualized in Figs. 1 and 2. The genes involved in the blue module were associated with the Rap1 signalling pathway, melanogenesis and holinergic synapses according to KEGG analysis, while the genes in of the turquoise module were associated with positive regulation of osteoblast differentiation and the ephrin receptor signaling pathway according to GO BP analysis, and the Ras signaling pathway, pathways in cancer and signaling pathways regulating the pluripotency of stem cells according to KEGG analysis. Theseresults are presented in Table II.

Survival analysis depending on the hub genes. In the dataset from TCGA database, the follow-up information for only
62 cases included the RFS. The prognostic value of $20 \mathrm{hub}$ genes was assessed using the survival package in R software. Kaplan-Meier analysis indicated that a higher expression of methyltransferase like 7B (METTL7B) was associated with a poorer RFS of EAC patients ( $\mathrm{P}=0.006$; Fig. 3).

CPH analysis. In the univariate CPH model, only the pathological TNM stage was marginally significantly associated with a higher risk of recurrence, while the pathological TNM stage $(\mathrm{P}=0.056)$ and the pathological $\mathrm{N}$ stage $(\mathrm{P}=0.140)$ were used to adjust the prognostic gene, METTL7B, in the multivariate $\mathrm{CPH}$ model (Table III). As an ordinal categorical variable, overexpressed METTL7B was significantly associated with a higher risk of recurrence. However, the pathological TNM stage and N stage weakened the association between METTL7B expression levels and RFS $(\mathrm{P}=0.092)$. As unordered categorical variables, Q3 and Q4 of METTL7B were significantly associated with a higher risk of recurrence in the univariate $\mathrm{CPH}$ model, while only Q3 of METTL7B was significantly associated with a higher risk of recurrence compared with that of the lowest quartile adjusted by the pathologic TNM stage and N stage (Table IV).

Validation of differential expression of prognostic genes. As displayed in Fig. 4, the METTL7B expression levels were 
Table II. Significantly enriched GO terms in the category biological process and Kyoto Encyclopedia of Genes and Genomes pathways in the different co-expression modules.

\begin{tabular}{|c|c|c|c|}
\hline Term or pathway & Description & Enriched genes (n) & Adjusted P-value \\
\hline \multicolumn{4}{|l|}{ Module blue } \\
\hline hsa04015 & Rap1 signaling pathway & 5 & $4.10 \times 10^{-2}$ \\
\hline hsa04916 & Melanogenesis & 4 & $4.28 \times 10^{-2}$ \\
\hline hsa04725 & Cholinergic synapse & 4 & $4.31 \times 10^{-2}$ \\
\hline hsa04540 & Gap junction & 4 & $4.46 \times 10^{-2}$ \\
\hline hsa05143 & African trypanosomiasis & 3 & $4.54 \times 10^{-2}$ \\
\hline hsa04071 & Sphingolipid signaling pathway & 4 & $4.59 \times 10^{-2}$ \\
\hline hsa04918 & Thyroid hormone synthesis & 4 & $4.61 \times 10^{-2}$ \\
\hline hsa04915 & Estrogen signaling pathway & 4 & $4.67 \times 10^{-2}$ \\
\hline hsa04270 & Vascular smooth muscle contraction & 4 & $4.82 \times 10^{-2}$ \\
\hline hsa04911 & Insulin secretion & 4 & $4.84 \times 10^{-2}$ \\
\hline \multicolumn{4}{|l|}{ Module turquoise } \\
\hline GO:0045669 & Positive regulation of osteoblast differentiation & 5 & $1.48 \times 10^{-2}$ \\
\hline GO:0048013 & ephrin receptor signaling pathway & 5 & $3.04 \times 10^{-2}$ \\
\hline hsa04014 & Ras signaling pathway & 9 & $3.09 \times 10^{-4}$ \\
\hline hsa05200 & Pathways in cancer & 10 & $7.90 \times 10^{-4}$ \\
\hline hsa04550 & $\begin{array}{l}\text { Signaling pathways regulating pluripotency of } \\
\text { stem cells }\end{array}$ & 7 & $1.05 \times 10^{-3}$ \\
\hline hsa05205 & Proteoglycans in cancer & 7 & $3.84 \times 10^{-3}$ \\
\hline hsa04810 & Regulation of actin cytoskeleton & 7 & $4.12 \times 10^{-3}$ \\
\hline hsa05166 & HTLV-I infection & 7 & $9.65 \times 10^{-3}$ \\
\hline hsa04360 & Axon guidance & 5 & $1.93 \times 10^{-2}$ \\
\hline hsa04015 & Rap1 signaling pathway & 6 & $1.99 \times 10^{-2}$ \\
\hline hsa04510 & Focal adhesion & 6 & $2.09 \times 10^{-2}$ \\
\hline hsa04310 & Wnt signaling pathway & 5 & $2.35 \times 10^{-2}$ \\
\hline hsa04151 & PI3K-Akt signaling pathway & 7 & $2.43 \times 10^{-2}$ \\
\hline
\end{tabular}

GO, Gene Ontology; hsa, Homo sapiens; PI3K, phosphoinositide-3 kinase; HTLV, human T-lymphotropic virus.

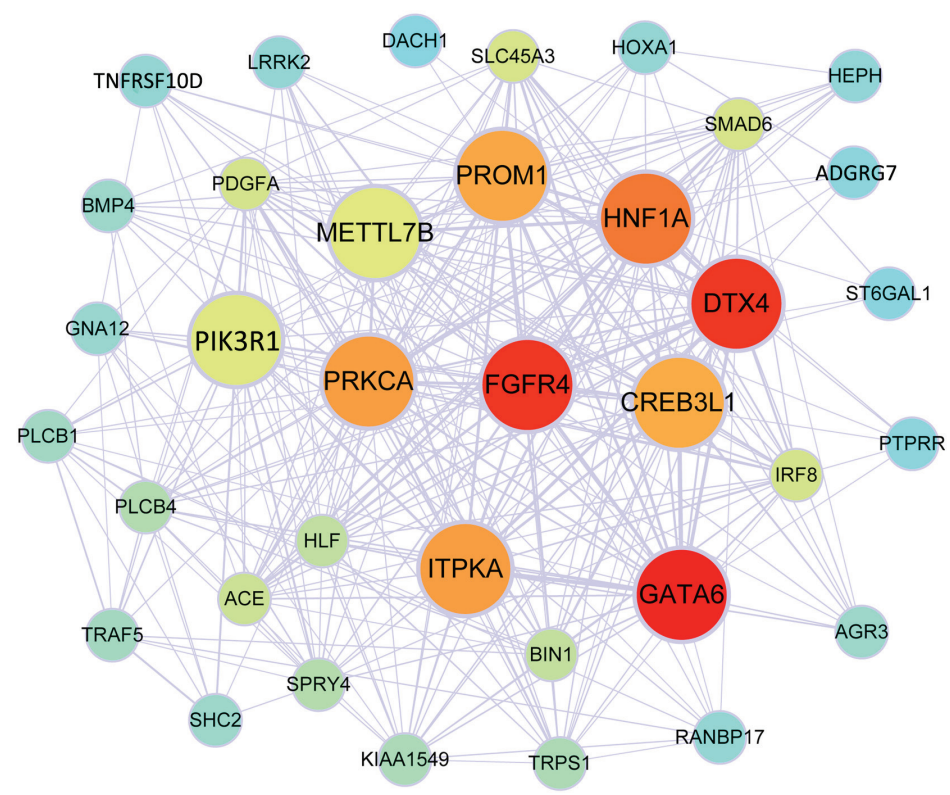

Figure 1. Co-expression network of the blue module identified by WGCNA. The colour spectrum illustrates the degree of connectivity of the nodes, with red indicating the highest degree of connectivity and turquoise the lowest degree of connectivity. The nodes with a larger size represent hub genes among the top 10 with the highest network connectivity degree. WGCNA, Weighted Gene Co-Expression Network Analysis. 


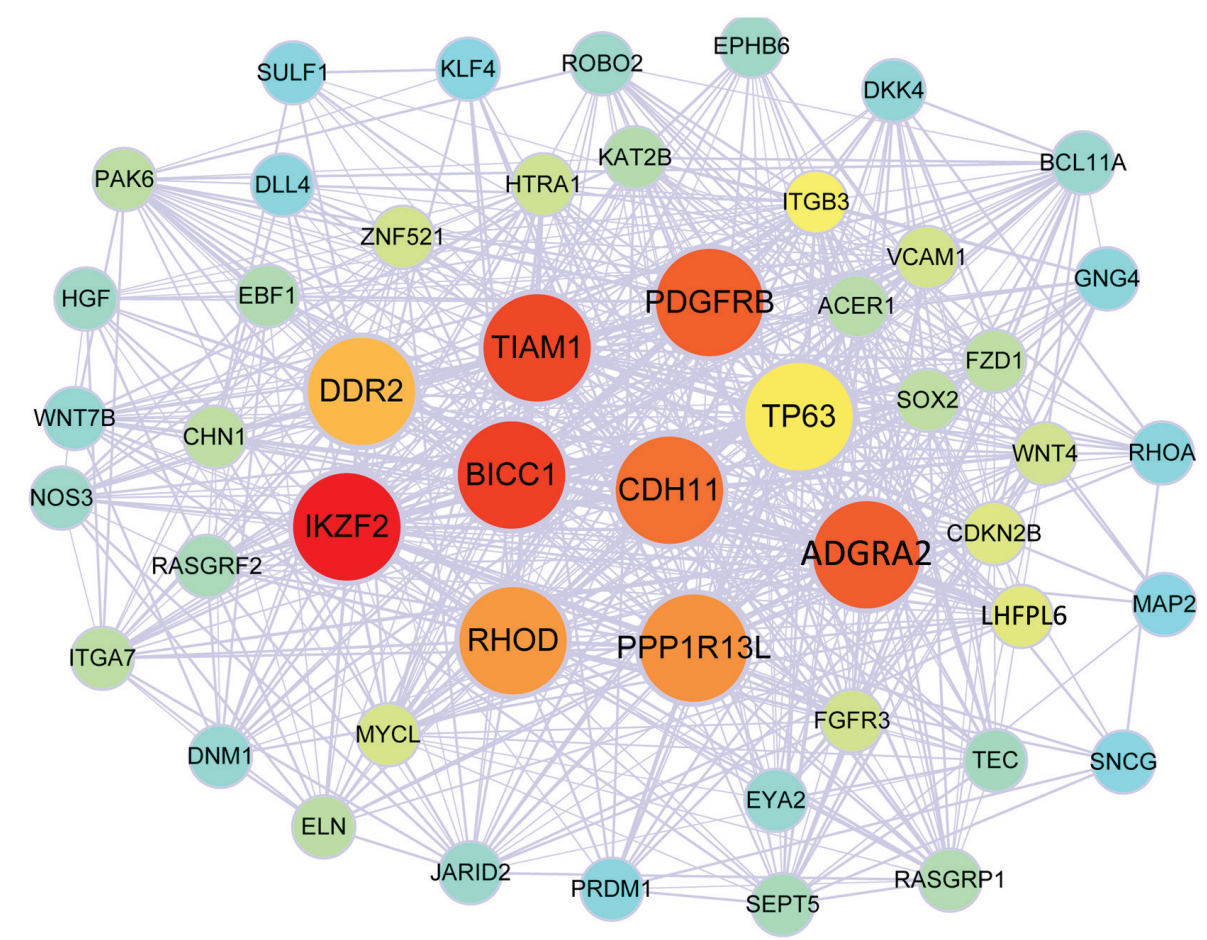

Figure 2. Co-expression network of the turquoise module identified by WGCNA. The colour spectrum illustrates the degree of connectivity of the nodes, with red indicating the highest degree of connectivity and turquoise the lowest degree of connectivity. The nodes with a larger size represent hub genes among the top 10 with the highest network connectivity degree. WGCNA, Weighted Gene Co-Expression Network Analysis.

significantly increased in EAC tissues compared with those in paracancerous lesions in the two additional datasets used for validation. The relative METTL7B expression levels in EAC vs. paired paracancerous lesions were $8.95 \pm 0.55$ vs. $4.58 \pm 0.42$ $(\mathrm{P}<0.0001)$ based on the data from the TCGA database and $8.66 \pm 0.25$ vs. $5.99 \pm 0.07(\mathrm{P}<0.0001)$ based on data from the GSE26886 dataset.

\section{Discussion}

In the present study, a total of 130 DEGs associated with EAC were identified, including 82 upregulated and 48 downregulated genes. The upregulated genes were associated with extracellular matrix organization, disassembly, PI3K-Akt, and the Rap1 and Ras signaling pathways, while the downregulated genes were associated with the Wnt signaling pathway. Among them, 2 co-expression modules and 20 hub genes were identified. The blue module was associated with the Rap1 signaling pathway, while the turquoise module was associated with the Ras and Rap1 signaling pathways. Subsequently, Kaplan-Meier analysis of hub genes revealed that higher expression of METTL7B was significantly associated with poorer RFS. The CPH model also confirmed this result. Finally, the overexpression of METTL7B in EAC tissues was also validated using data from TCGA and another microarray dataset, GSE26886. Therefore, METTL7B may be regarded as a key gene, while the Ras and Rap1 signaling pathways may be crucial mechanisms of genesis of EAC. In addition, all enriched GO BPs and KEGG pathways may participate in mechanisms underlying EAC progression and require to be considered in future studies.

METTL7B is a protein-coding gene located at chromosome $12 \mathrm{q} 13.2$. It was reported to be a major component of

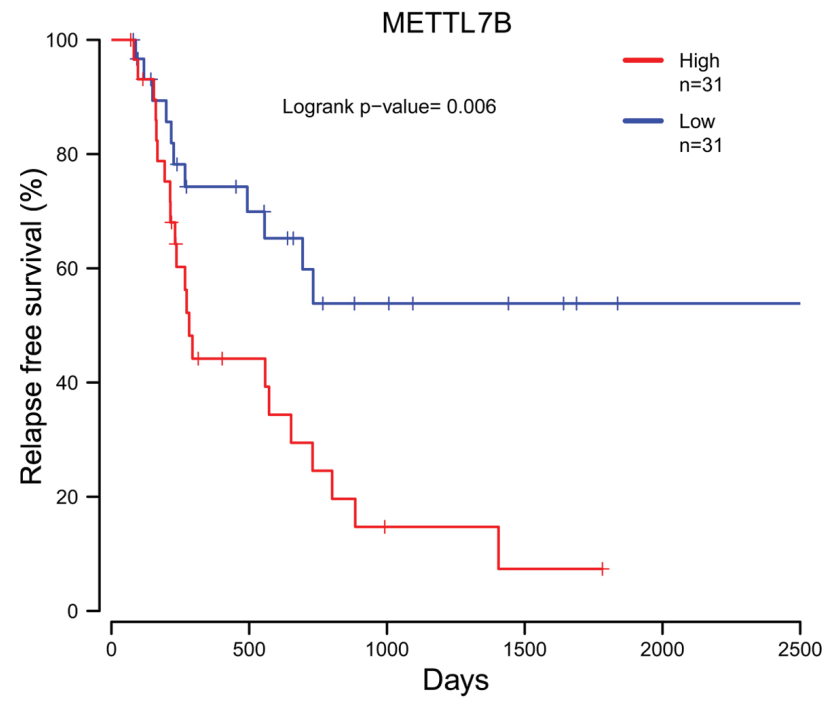

Figure 3. Prognostic value of METTL7B for relapse-free survival of EAC patients. EAC patients were divided into low and high expression groups according to the median expression level. EAC, esophageal adenocarcinoma; METTL7B, methyltransferase like 7B.

hepatic lipid droplets and a prognostic marker of non-alcoholic steatohepatitis (18). Although certain studies have revealed that METTL7B is associated with common lipid metabolism and functional organelle production, its precise functions have been rarely reported $(19,20)$. A recent study focusing on breast cancer cell lines identified that downregulation of METTL7B, which may be caused by a reduction in Rho-related BTB domain-containing protein 1 , leads to a profound fragmentation of the Golgi apparatus; this then contributes to a loss 
Table III. Univariate Cox proportional hazard regression analysis of parameters affecting relapse-free survival.

\begin{tabular}{lccc}
\hline Parameter & $\mathrm{n}$ & HR (95\% CI) & P-value \\
\hline Age ( $\leq 65$ vs. $>65$ years) & $29: 32$ & $0.93(0.47-1.83)$ & 0.825 \\
History of alcohol use (yes vs. no) & $10: 50$ & $0.59(0.25-1.36)$ & 0.215 \\
History of Barrett's esophagus (yes vs. no) & $37: 17$ & $1.03(0.48-2.22)$ & 0.936 \\
Location of lesion (mid vs. distal) & $5: 56$ & $0.73(0.21-2.45)$ & 0.605 \\
Pathologic N-stage (0/1 vs. 2/3) & $38: 9$ & $2.07(0.79-5.43)$ & 0.140 \\
Pathologic T-stage (1/2 vs. 3/4) & $26: 21$ & $1.41(0.59-3.37)$ & 0.435 \\
Pathologic TNM stage (1/2 vs. 3/4) & $26: 18$ & $2.37(0.98-5.72)$ & 0.056 \\
\hline
\end{tabular}

HR, hazard ratio; CI, confidence interval.

Table IV. Risk of recurrence for each quartile of methyltransferase like 7B expression levels.

\begin{tabular}{|c|c|c|c|c|c|}
\hline Calculation & Q1 & Q2 & Q3 & Q4 & P-value for trend \\
\hline Range & $6.39-8.17$ & $8.18-9.01$ & $9.04-9.49$ & $9.5-10.62$ & \\
\hline Patients, $\mathrm{n}$ & 16 & 15 & 14 & 16 & \\
\hline Model 1, HR (95\% CI) & Ref & $2.78(0.74-10.53)$ & $6.46(1.78-23.38)$ & $4.16(1.16-14.96)$ & \\
\hline P-value & & 0.131 & 0.005 & 0.029 & 0.013 \\
\hline (No. of patients) & $(n=13)$ & $(n=11)$ & $(n=7)$ & $(n=12)$ & \\
\hline Model 2 (HR (95\% CI)) & Ref & $2.18(0.52-9.12)$ & $4.62(1.08-19.75)$ & $2.9(0.76-11.08)$ & \\
\hline P-value & & 0.287 & 0.039 & 0.12 & 0.092 \\
\hline
\end{tabular}

Range, expression level range in each quartile; Model 1, crude data with no adjustment; Model 2, adjustment for pathological TNM stage and $\mathrm{N}$ stage; Q, quartile. P-value for trend, P-value of cox proportional hazard regression analysis of METTL7B expression level transformed into ordered categorical variable affecting relapse-free survival. HR, hazard ratio; CI, confidence interval. HR (95\% CI), HR and CI compared with Q1 group as METTL7B expression level transformed into unordered categorical variable. Ref, reference.
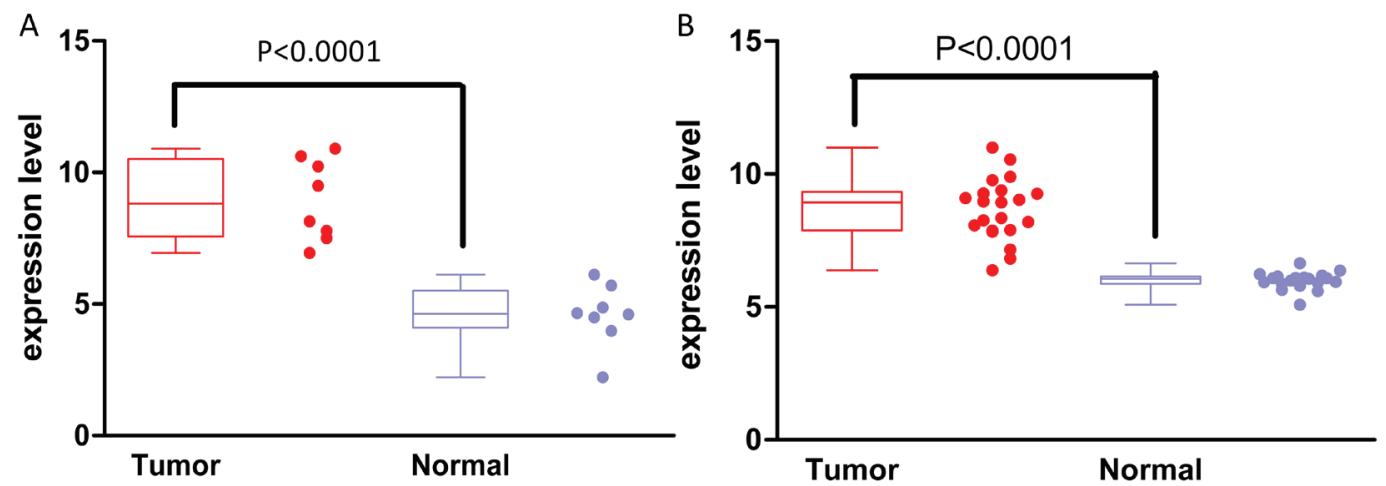

Figure 4. Validation of differential expression of METTL7B in EAC. (A) Expression level of METTL7B in EAC ( $\mathrm{n}=8$ ) and normal epithelium tissues ( $\mathrm{n}=8$ ) based on data from The Cancer Genome Atlas. (B) Expression level of METTL7B in EAC ( $\mathrm{n}=21)$ and normal epithelium tissues ( $\mathrm{n}=19)$ based on data from the microarray dataset GSE26886. In the box plot, the boxes indicate the median and interquartile range of data, while the error bars represent the minimum and maximum values. The data points display the exact numerical expression level for each sample. EAC, esophageal adenocarcinoma; METTL7B, methyltransferase like 7B.

of normal epithelial polarity and finally converts mammary epithelial cell phenotypes into an invasive one (21). However, to the best of our knowledge, no previous study has assessed the role of METTL7B in esophageal neoplasms. Therefore, the potential existence of a mechanism of this specific gene in EAC analogous to that in breast cancer is worthy of investigation.
The Ras signaling pathway is one of the most thoroughly studied pathways in various types of human cancer, including EAC. The mitogen-activated protein kinase (MAPK) signaling pathway, a key downstream effector of Ras signaling, is shared by four distinct cascades named as extracellular signal-regulated kinase (ERK)1/2, c-Jun N-terminal 
kinase (JNK), p38MAPK and ERK5. Upregulation of the Ras signaling pathway activates the MAPK pathway, and this or other dysregulations in downstream signaling probably lead to carcinogenesis. The ERK1/2 pathway has a crucial role in regulating cellular growth and differentiation. Accordingly, Fan et al (22) demonstrated that inhibition of ERK activation prevented proliferation of human esophageal cancer cells by inducing cell cycle arrest in G0/G1 phase. The p38MAPK and JNK pathways are closely linked to stress, including inflammation and apoptosis. It was observed that Ras-JNK signaling significantly enhances the invasive behaviour of EAC cell lines (23), while Connor et al (24) indicated that JNK activation leads to the induction of apoptosis of EAC cells. A similar phenomenon was reported for Ras-p38MAPK signalling $(25,26)$, and it may therefore be hypothesized that these two pathways have a tumor suppressor or oncogenic role depending on certain conditions, e.g. the stage of EAC. From the above information, it is apparent that the Ras signaling pathway has a definite role in EAC, and therefore, the proteins associated with this signal transduction pathway that were identified in the present bioinformatics analysis are worthy of further investigation.

The Rap1 pathway is another 'hot spot' of cancer research. Rap1 is a member of the Ras-like small GTPase family with two subtypes, Rapla and Rap1b. The Rap1 signaling pathway is known to be over-activated in various tumor types. Furthermore, it has been reported to promote tumor cell migration, invasion and metastasis in the development of cancer, including, prostate cancer (27), head and neck cancer (28) and esophageal squamous cell carcinoma (29). Conversely, active Rap1 also prevents tumor invasion and metastasis in the bladder, lung and brain (29). However, to the best of our knowledge, the role of the Rapl signaling pathway in EAC has remained unreported. Further experiments are required to verify the role of the Rap1 signaling pathway and EAC.

In conclusion, in the present study, a total of 130 DEGs were identified in EAC, from which 2 co-expression modules were established. The enriched pathways, including the Ras and Rap1 signaling pathways, may be closely linked to EAC progression. Furthermore, METTL7B may be a key gene associated with the prognosis of EAC. However, no additional experiments or clinical research were performed to verify these results. Thus, further studies are required, which should focus on a more detailed clinical application of this key gene and associated pathways.

\section{Acknowledgements}

Not applicable.

\section{Funding}

The present study was supported by National Natural Science Foundation of China (grant nos. 81570484, 81600424 and 81502041).

\section{Availability of data and materials}

Not applicable.

\section{Authors' contributions}

ZD conducted the research, analysed the data and wrote this manuscript. SX designed the study, performed the research and revised the paper. JW performed the research and wrote this manuscript. HZ, TZ and YC performed the research and acquired the data.

\section{Ethical approval and consent to participate}

Not applicable.

\section{Patient consent for publication}

Not applicable.

\section{Competing interests}

The authors declare that they have no competing interests.

\section{References}

1. Domper Arnal MJ, Ferrández Arenas Á and Lanas Arbeloa Á: Esophageal cancer: Risk factors, screening and endoscopic treatment in Western and Eastern countries. World J Gastroenterol 21: 7933-7943, 2015.

2. Siegel R, Naishadham D and Jemal A: Cancer statistics, 2012. CA Cancer J Clin 62: 10-29, 2012.

3. Brown CS, Gwilliam N, Kyrillos A, Lutfi W, Lapin B, Kim KW, Krantz SB, Howington JA, Yao K and Ujiki MB: Predictors of pathologic upstaging in early esophageal adenocarcinoma: Results from the national cancer database. Am J Surg 216: 124-130, 2018.

4. Chen M, Huang J, Zhu Z, Zhang J and Li K: Systematic review and meta-analysis of tumor biomarkers in predicting prognosis in esophageal cancer. BMC Cancer 13: 539, 2013.

5. Darlavoix T, Seelentag W, Yan P, Bachmann A and Bosman FT: Altered expression of CD44 and DKK1 in the progression of Barrett's esophagus to esophageal adenocarcinoma. Virchows Arch 454: 629-637, 2009.

6. Smith CM, Watson DI, Michael MZ and Hussey DJ: MicroRNAs, development of Barrett's esophagus, and progression to esophageal adenocarcinoma. World J Gastroenterol 16: 531-537, 2010.

7. Leidner RS, Ravi L, Leahy P, Chen Y, Bednarchik B, Streppel M, Canto M, Wang JS, Maitra A, Willis J, et al: The MicroRNAs, MiR-31 and MiR-375, as candidate markers in Barrett's esophageal carcinogenesis. Genes Chromosomes Cancer 51: 473-479, 2012.

8. Barrett T, Wilhite SE, Ledoux P, Evangelista C, Kim IF, Tomashevsky M, Marshall KA, Phillippy KH, Sherman PM, Holko M, et al: NCBI GEO: Archive for functional genomics data sets-update. Nucleic Acids Res 41: D991-D995, 2013.

9. Wang Z, Cheng Y, Abraham JM, Yan R, Liu X, Chen W, Ibrahim S, Schroth GP, Ke X, He Y and Meltzer SJ: RNA sequencing of esophageal adenocarcinomas identifies novel fusion transcripts, including NPC1-MELK, arising from a complex chromosomal rearrangement. Cancer 123: 3916-3924, 2017.

10. Ritchie ME, Phipson B, Wu D, Hu Y, Law CW, Shi W and Smyth GK: limma powers differential expression analyses for RNA-sequencing and microarray studies. Nucleic Acids Res 43: e47, 2015.

11. Ashburner M, Ball CA, Blake JA, Botstein D, Butler H, Cherry JM, Davis AP, Dolinski K, Dwight SS, Eppig JT, et al: Gene Ontology: Tool for the unification of biology The gene ontology consortium. Nat Genetics 25: 25-29, 2000.

12. Kanehisa M and Goto S: KEGG: Kyoto encyclopedia of genes and genomes. Nucleic Acids Res 28: 27-30, 2000.

13. Huang DW, Sherman BT, Tan Q, Collins JR, Alvord WG, Roayaei J, Stephens R, Baseler MW, Lane HC and Lempicki RA: The DAVID gene functional classification tool: A novel biological module-centric algorithm to functionally analyze large gene lists. Genome Biol 8: R183, 2007.

14. Langfelder P and Horvath S: Fast R functions for robust correlations and hierarchical clustering. J Stat Softw 46: i11, 2012. 
15. Shannon P, Markiel A, Ozier O, Baliga NS, Wang JT, Ramage D, Amin N, Schwikowski B and Ideker T: Cytoscape: A software environment for integrated models of biomolecular interaction networks. Genome Res 13: 2498-2504, 2003.

16. Lin $\mathrm{H}$ and Zelterman D: Modeling survival data: Extending the cox model. J Am Stat Assoc 44: 85-86, 2002.

17. Wang Q, Ma C and Kemmner W: Wdr66 is a novel marker for risk stratification and involved in epithelial-mesenchymal transition of esophageal squamous cell carcinoma. BMC Cancer 13: 137, 2013.

18. Thomas A, Klein MS, Stevens AP, Reinders Y, Hellerbrand C, Dettmer K, Gronwald W, Oefner PJ and Reinders J: Changes in the hepatic mitochondrial and membrane proteome in mice fed a non-alcoholic steatohepatitis inducing diet. J Proteomics 80: 107-122, 2013.

19. Zehmer JK, Bartz R, Liu P, Anderson RG: Identification of a novel $\mathrm{N}$-terminal hydrophobic sequence that targets proteins to lipid droplets. J Cell Sci 121: 1852-1860, 2008.

20. Wu CC, MacCoss MJ, Mardones G, Finnigan C, Mogelsvang S, Yates JR 3rd and Howell KE: Organellar proteomics reveals golgi arginine dimethylation. Mol Biol Cell 15: 2907-2919, 2004

21. McKinnon CM and Mellor H: The tumor suppressor RhoBTB controls Golgi integrity and breast cancer cell invasion through METTL7B. BMC Cancer 17: 145, 2017.

22. Fan W, Sun L, Zhou JQ, Zhang C, Qin S, Tang Y, Liu Y, Lin SS and Yuan ST: Marsdenia tenacissima extract induces G0/G1 cell cycle arrest in human esophageal carcinoma cells by inhibiting mitogen-activated protein kinase (MAPK) signaling pathway. Chin J Nat Med 13: 428-437, 2015.

23. Onwuegbusi BA, Rees JR, Lao-Sirieix P and Fitzgerald RC: Selective loss of TGF beta smad-dependent signalling prevents cell cycle arrest and promotes invasion in oesophageal adenocarcinoma cell lines. Plos One 2: e177, 2007.
24. Connor CA, Adriaens M, Pierini R, Johnson IT and Belshaw NJ: Procyanidin induces apoptosis of esophageal adenocarcinoma cells via JNK activation of c-Jun. Nutr Cancer 66: 335-341, 2014.

25. Sutter AP, Maaser K, Barthel B and Scherübl H: Ligands of the peripheral benzodiazepine receptor induce apoptosis and cell cycle arrest in oesophageal cancer cells: Involvement of the p38MAPK signalling pathway. Br J Cancer 89: 564-572, 2003.

26. Hu JL, Xiao L, Li ZY, Wang Q, Chang Y and Jin Y: Upregulation of HO-1 is accompanied by activation of p38MAPK and mTOR in human oesophageal squamous carcinoma cells. Cell Biol Int 37: 584-592, 2013.

27. Menon J, Doebele RC, Gomes S, Bevilacqua E, Reindl KM and Rosner MR: A Novel interplay between Rap1 and PKA regulates induction of angiogenesis in prostate cancer. Plos One 7: e49893, 2012.

28. Banerjee R, Russo N, Liu M, Van Tubergen E and D'Silva NJ: Rap1 and its regulatory proteins: The tumor suppressor, oncogene, tumor suppressor gene axis in head and neck cancer. Small GTPases 3: 192-197, 2012.

29. Zhang YL, Wang RC, Cheng K, Ring BZ and Su L: Roles of Rap1 signaling in tumor cell migration and invasion. Cancer Biol Med 14: 90-99, 2017.

This work is licensed under a Creative Commons Attribution-NonCommercial-NoDerivatives 4.0 International (CC BY-NC-ND 4.0) License. 